\title{
Contactless Fingerprint Recognition and Fingerprint Spoof Mitigation using CNN
}

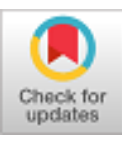

\author{
Sunil B Nirmal, Kishor S Kinage
}

\begin{abstract}
Contactless identification of fingerprints has gained considerable attention as it can offer more hygienic and accurate personal identification. Despite these benefits, contactless $2 D$ imagery often leads to partial $2 D$ fingerprints as it requires relatively higher user cooperation during contactless $2 D$ imagery. This paper develops a CNN framework for recognizing contactless fingerprint images-based on database. Our framework uses fingerprint minutiae and particular ridge map region to train a CNN first. Over several popular deep learning, our experiments presented in this paper achieve good results with greater accuracy. Experimental results obtained in this paper shows the effectiveness of the proposed approach and illustrate a significant improvement in methods of fingerprint recognition. The proposed work also helps to mitigate spoofing of fingerprints, thus providing greater security.
\end{abstract}

Keywords: Bio-metric, Neural network, CNN, Authentication, Spoofing.

\section{INTRODUCTION}

A uthentication technologies are used in security awareness in many places, with increasing financial activities. Traditional authentication such as passwords, personal identification numbers, smart cards were largely unable to meet convenience, reliability and security requirements in a wide variety of applications. Face recognition is the most common of these techniques; fingerprint recognition, voice recognition and iris recognition are readily available even for smartphone users. For civil and law enforcement applications around the world, contact-based 2D fingerprint identification is widely used. Such traditional methods for acquisition of fingerprint images by pressing finger against the surface that are hard (such as, silicon, glass, polymer, etc) can often lead to a partial or degraded images due to placement of improper fingers. As a result, full fingerprint potential is not realized. Hygiene is serious issue in contact-based system. To address issues mentioned in above technique, touchless finger imaging systems have emerged in the recent years. As unique data is available from fingerprint images, such approach can be used in getting accurate personal identification. The minutiae features are widely regarded by law enforcement experts and commercial 2D fingerprint systems available today as being the most reliable and widely used.

Manuscript published on November 30, 2019

* Correspondence Author

Sunil B Nirmal*, currently a final year M Tech student of Pimpri Chinchwad College of Engg. Pune University, India.

Dr.Kishor Kinage, professor at Pimpri Chinchwad college of engg, Pune, India.

(C) The Authors. Published by Blue Eyes Intelligence Engineering and Sciences Publication (BEIESP). This is an open access article under the CC-BY-NC-ND license http://creativecommons.org/licenses/by-nc-nd/4.0/
Tasks such as recovery, representation, selection, and matching of $2 \mathrm{D}$ fingerprints is essentially a problem.This paper provides algorithmic details on matching and spoofing of $2 \mathrm{D}$ fingerprints.

A convolutional neural network is used for the feature extraction as well as classification. Once the image is preprocessed, it is sent for edge detection and finally to $\mathrm{CNN}$. There is no need to use separate technique for feature extraction, as CNN is smart enough to compute the feature from given image. This system can be used in various application that can promote to more security as we have added feature to mitigate fingerprint spoof. A fingerprint spoof is a severe threat to anyone's authorization.

\section{THEORY}

\section{A. LITERATURE SURVEY}

The paper[3] investigated a approach to match contactless fingerprint images with contact-based images. Respective approach is based on a multi-Siamese convolutionary neural network whose architecture emphasizes for matching to generate deep feature representations.

This paper [5] investigates contactless identification of fingerprint images acquired using webcam. The acquired images are firstly subjected to robust preprocessing steps to extract region of interest and normalize uneven illumination. Texture features are used to achieve reliable identification.It achieved average recognition accuracy of $93.97 \%$ and equal error rate of $3.95 \%$ from two session images.

In [16], Optical and Capacitive sensors were used. The average deformation model is used to distort the minutiae points of images acquired using one sensor before comparing them with the minutiae points of images corresponding to another sensor. A significant performance improvement is observed when the proposed scheme is utilized to compare fingerprint images originating from two different sensors

This paper [17] investigates an approach for 3D fingerprint identification using recovered surface normal information. The rank-one recognition accuracy is $90.1 \%$. In [9], a technique for creating and identifying a fingerprint spoof image is described. Here, a center patch region is considered and various operations are performed on this region. Some ridges on this patch are missing and this missing part can be used to detect spoof fingerprint.

\section{B. SYSTEM DIAGRAM}

The fundamental steps for the contactless fingerprint recognition system are shown in the form of blocks in figure 1 below. It's a system that takes finger images without any physical contact. 


\section{Contactless Fingerprint Recognition and Fingerprint Spoof Mitigation using CNN}

The images can be acquired by using high resolution camera. We use database that contains a contactless fingerprint. For training purpose, we passed multiple images that are processed and stored in database. But for testing purpose we passed single image at a time.

The proposed system for fingerprints consists of units such as: pre-processing, features extraction and matching. The passed image is first preprocessed before going to fingerprint feature extraction and matching.

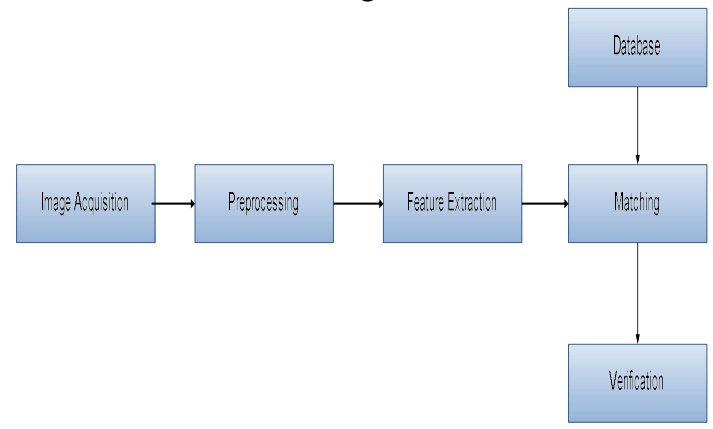

Fig 1: Steps of Fingerprint Recognition System

\section{Database A:}

A database consist of 275 image collection of 55 different people. Out of 55 person, we provide authorization for eleven person and remaining are unauthorized. Out of 11 authorized people, seven are male candidates while four are female candidates. We used contactless fingerprint images available on GIT-HUB.

\section{Database B:}

We modify the database A to create a database B which contains the spoof images. The spoof image are created using operations such as resizing, translation and rotation.

\section{Fingerprint Acquisition:}

Image is read from a database. Database contains nearly about 275 images from 55 different people. 5 different images of each person are stored in database.

\section{Preprocessing:}

This is the system's initial step. A fingerprint image acquired can be of different size. So, image is generally resized to some fixed size. It can also happen that image acquired can be of low quality that can have low contrast between valleys and ridges. Since the image acquisition stage is not always monitored to only accept high-quality images, fingerprint image enhancement and noise reduction are important pre-processing factors in the accurate detection of fingerprint.
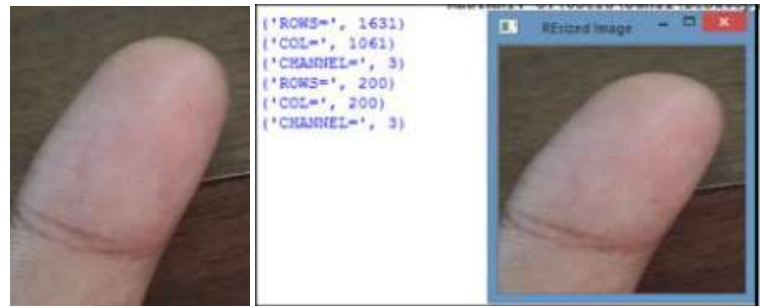

Fig 2: Before resize and after resize

We can improve the image quality by cropping the region of the fingerprint in the image and then performing histogram equalization in order to increase the clear information.

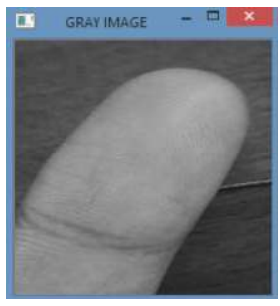

Fig 3: Image in Gray

Histogram Equalization creates very good image enhancement in a very good fashion due to which image intensities are distributed properly. After resizing, it might happen that color image maynot be suitable for edge detection. So, we convert given RGB color image into gray form. A gray form of image is shown in figure. A gray form image is easier for edge detectors to find accurate egdes in image. We also take care that all the pixels are uniformly distributed. If not so, then we adjust contrast and make histogram equalization.
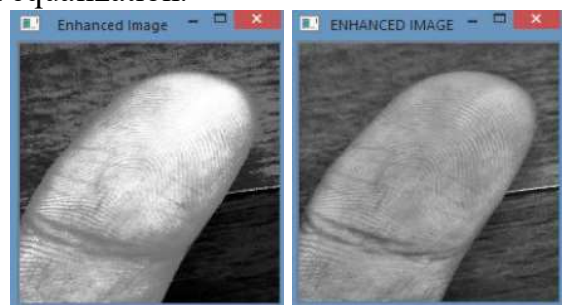

Fig 4: Image with contrast adjustmented.

This are the sub-steps that are carried out in pre-processing step.
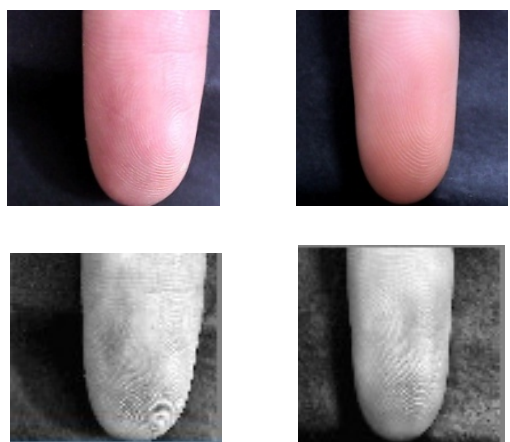

Fig 5: Contactless fingerprint and respective preprocessed contactless fingerprint from dataset.

Edge Detection:

Edge detection of image is important before extracting features. We used "CANNY" algorithm for detecting edges. Tensorflow has made much of the work simple in machine learning. By using function: 'cv2.canny', its much easy to apply canny edge detector to image.

An edge in an image may point in a variety of directions, so the Canny algorithm uses four filters to detect horizontal, vertical and diagonal edges in the image. The edge detection operator (such as Roberts, Prewitt, or Sobel) returns a value for the first derivative in the horizontal direction $\left(\mathrm{G}_{x}\right)$ and the vertical direction $\left(\mathrm{G}_{y}\right)$. From this the edge gradient can be determined: 


$$
G=\sqrt{G x^{2}+G y^{2}}
$$

The characteristics of an image are classified as follows:

(1) Special features like Colour, gradient, parameter spectrum, etc.

(2) Geometric features like Edge, shape, size and so on.

(3) Textural features like pattern, spatial frequency, homogeneity etc.

Matching: Depending on the obtained matching score two fingerprints are declared as authorized or unauthorized, the two Fingerprint images are matched using extracted local features.

\section{CNN ALGORITHM}

CNN's network design consists of multiplelayers mentioned below. Convolutional neural networks are made up of different layers between the layers of input and output. These layers, known as the hidden layers, consist mainly of the Convolutional layer, Pooling layer and Fullly connected layer. In our model, we used Convolutional layer and pooling layer.

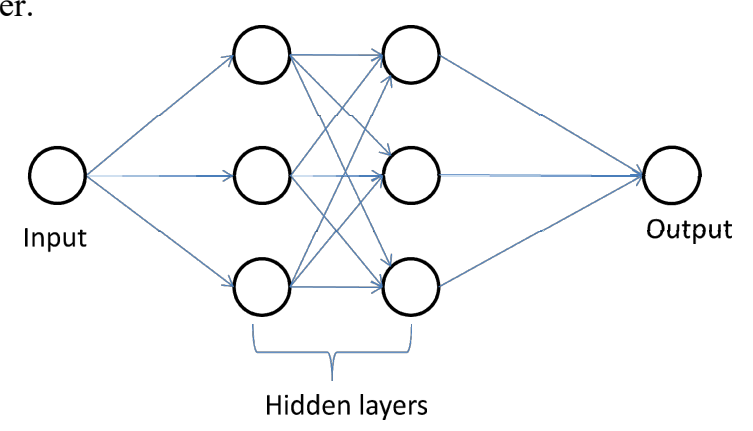

Fig 6: Structure of CNN

\section{Convolutional Layer}

Each convolutional neural network consists of multiple layers of convolution depending on the requirements of the network. The first convolutional layers are responsible for learning relatively low - level features such as with edges, corners, etc. These layers ' output is often fed to other convolutionary layers that learn features of a higher level.Each neuron in this layer is connected in the preceding layer to only a limited number of neurons. The number of neurons in which they are connected is known as the convolutionary layer's receptive field. These layers consist of a filter with a $3 \times 3$ size. During the forward pass, the filter is slid (or more accurately converted) across the width and height of the input volume and the point products are calculated between the filter entries and the input position of the final feature map.Multiple filters are used for convolution, however, as images have multiple features, meaning the depth of the feature maps (which is a hyper parameter) equal to the number of filters used.

\section{Pooling Layer:}

To reduce the number of parameters, the pooling layer is used to decrease the spatial size or image resolution, thus reducing the computational burden.This is done by reducing the number of connections between the convolutionary layers. Usually they alternate between the convolutionary layers. Maximum pooling and average pooling are the most common types of pooling. Although the use of average pooling has been significantly reduced recently, max pooling remains one of the most common methods. Lp pooling is a

biologically inspired process from complex cells of visual cortex.

It is a method of pooling inspired by dropout. It selects a random activation within each pooling region based on a multinomial distribution instead of the maximum value.like in max - pooling, which ensures that non - maximum activations can also be used in feature maps. Mixed pooling is form of pooling that is the combination of average and max pooling.

A CNN is trained by passing multiple images at a time to it. Here in this approach, we passed 10 images at a time to $\mathrm{CNN}$ for training purpose. That is a batch size $=10$. Training of CNN is performed by making iterations of its layers. We made iteration of four. After four iterations, the training accuracy comes to be about 100 percent. Result of CNN training is shown in figure.

A CNN technique requires less pre-processing as compared to other classifier techniques. It is just because the number of parameters generated by $\mathrm{CNN}$ is less. CNN does not bother of image size. Its upto our approach of selecting filter size. If filter size is selected to be $5 \times 5$, then almost 25 parameters are created.

The training result is shown in figure below. We represented the training accuracy with respect to epoch in bar graph. We train the CNN till it gives 100 percent accuracy.

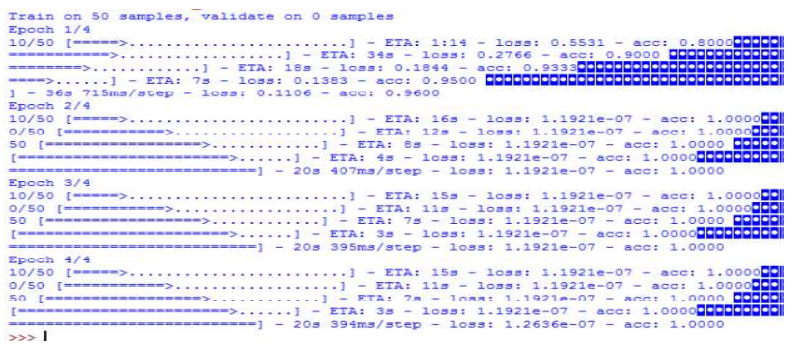

Fig 7: Training of CNN.

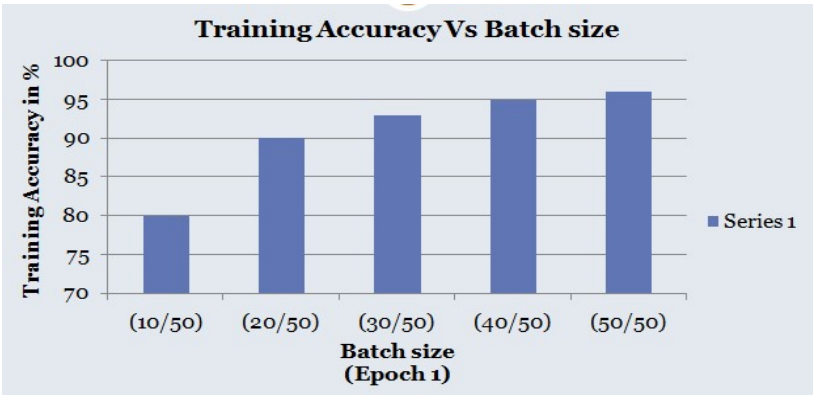

Fig 8:Training accuracy Vs Batch size(for epoch 1)

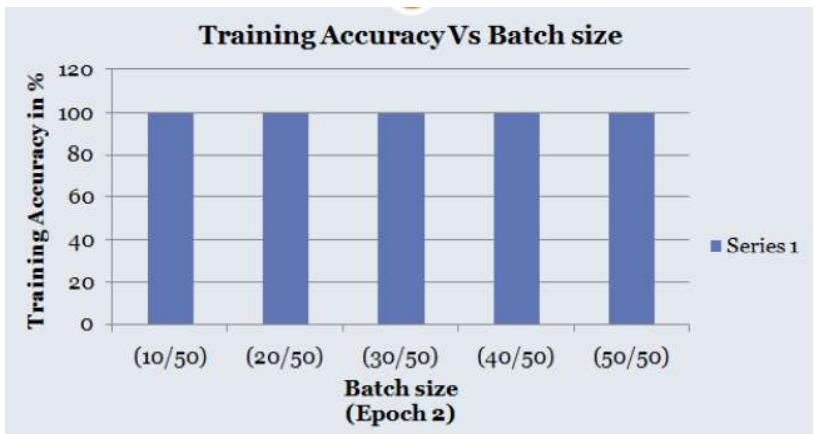

Fig 9:Training accuracy Vs Batch size(for epoch 2) 


\section{Contactless Fingerprint Recognition and Fingerprint Spoof Mitigation using CNN}

\section{Matching:}

Once the features are extracted, then as per features the weight scores are generated by $\mathrm{CNN}$. Depending on this weights, the matching of image is done. CNN predicts the score from each image that are passed for testing and for given threshold ( $t h=1$ in our case) $\mathrm{CNN}$ performs matching. We consider different threshold values for experimentation. Depending on outcomes, we represent the accuracy with respect to threshold values in the form of graph.(as shown in fig 11).

\section{Approach for fingerprint spoof mitigation:}

System designed has important advantage for mitigating spoof fingerprint. In this model, we applied some basic steps in creating database of fingerprint spoof images derived from original image. Steps used to make spoof image database are as follows:

Resizing: This is the basic step that can be applied for creating a spoof image.

We have resized given image into $100 \times 100$ form.

Translation:

Convert image into some missing lines(ridges).

Rotation:

Rotate image w.r.t rows and columns.

This is the possible attacks that occur on original fingerprint image when doing spoofing. So, we get a database of spoof images. So, when we pass such spoof image, then model will predict it as an unknown image. The sample images of finger spoofs are shown in figure 10.
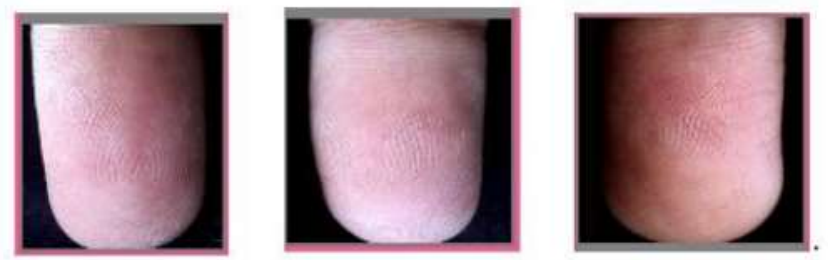

Fig.10 : Spoof Images

\section{RESULT AND DISCUSSION}

The results of experiment are shown and discussed below.

\begin{tabular}{|c|c|c|}
\hline & REC & NOT RE \\
\hline REC & $\begin{array}{l}\text { TP } \\
93\end{array}$ & $\begin{array}{l}\mathrm{FN} \\
7\end{array}$ \\
\hline NOT REC & $\begin{array}{c}\text { FP } \\
0\end{array}$ & $\begin{array}{l}\mathrm{TN} \\
40\end{array}$ \\
\hline
\end{tabular}

Fig.11:Confusion Matrix

For testing purpose, we used 140 images out of 275 images. accuracy $=T P+T N T P+F P+T N+F N$

For threshold $=1$, accuracy $=93+40140=0.95$

For threshold $=0.5$, accuracy $=60+31140=0.65$

For threshold $=1.5$, accuracy $=65+37140=0.728$

Accuracy with respect to different thresholds is shown graphically below:

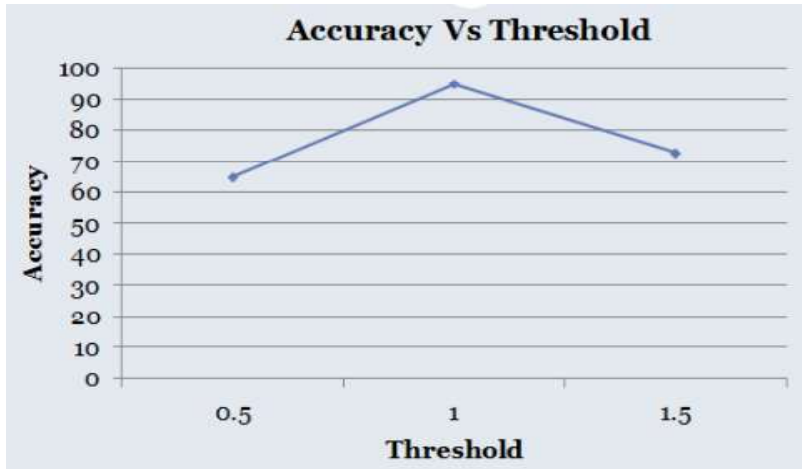

Fig.12: Accuracy Vs Threshold values.

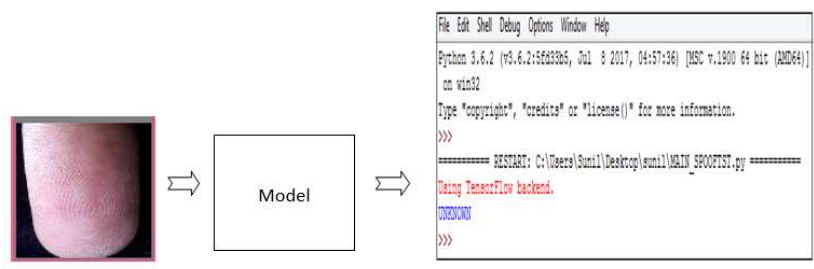

Fig.13: Output of model when spoof image is passed.

\section{CONCLUSION}

This paper proposes a CNN-based model for identification of contactless fingerprint recognition. A model is tested which achieved more accurate identification.Higher accuracy of recognition, low complexity and low storage requirements can make it popular for deployment. Accuracy is calculated by considering different threshold values. By experiments, for threshold $=1$, accuracy was seen maximum. Although the proposed CNN-based approach achieves good performance for contactless fingerprint recognition, it is still possible to improve identification accuracy by incorporating greater training set as well as training strategies. A fingerprint spoofing mitigation is important aspect of this model. System thus provides more security. By passing spoof image to model, it is detected as an unknown image as shown in figure.

\section{REFERENCES}

1. G. O. Young, "Synthetic structure of industrial plastics (Book style with paper title and editor)," in Plastics, 2nd ed. vol. 3, J. Peters, Ed. New York: McGraw-Hill, 1964, pp. 15-64.

2. M.Srinath\&P.Pakutharivu, "A Comprehensive Survey on Fingerprin Recognition Systems, Indian Journal of Science and Technology, 2015.

3. NeerajBharagava, AnchalKumawat, RituBharagava (2015) "Fingerprint Matching of Normalized Image based on Euclidean Distance". International Journal of computer Application .Volume 120-No 24.

4. Chenhao Lin and Ajay Kumar ,"A CNN-based Framework for Comparison of Contactless to Contact-based Fingerprints ", IEEE Transactions on Information Forensics and Security,2018.

5. R.Donidalabati, A. Genovese, V. Piuri and F. Scotti,'Touchless Fingerprint Biometrics: A survey on 2D and 3D Technologies", in journal of internet technology,2014.

6. A. Kumar and Y. Zhou, "Contactless fingerprint identification using level zero features," in Computer Vision and Pattern Recognition Workshops (CVPRW), 2011 IEEE Computer Society Conference on. IEEE, 2011, pp. 114-119.

7. P.Krishnasamy, S.Belongie and D.Kriegman, "Wet fingerprint recognition: Challenges and opportunities," in Biometrics (IJCB), International Joint Conference on. IEEE, 2011, pp. 1-7. 
8. DavideMaltoni, Dario Maio, Anil $\mathrm{K}$ Jain and SalilPrabhakar, "Handbook of Fingerprint Recognition", second edition Springer.

9. KarenSimonyan Andrew Zisserman,"Two-Stream Convolutional Networks for Action Recognition in Videos", Neural Information Processing Systems,2014,Vol1,pp.568-5.

10. TarangChugh and Anil K. Jain, "Fingerprint Spoof Buster: Use of Minutiae-centered Patches", Transactions On Information Forensics And Security 2018.

11. Weblink for downloading contactless fingerprint images used in this paper, https://github.com/Chenhao03/DATASET.

12. Y.-L. Boureau, J. Ponce, and Y. LeCun, "A theoretical analysis of feature pooling in visual recognition," in Proceedings of the 27th international conference on machine learning (ICML-10), 2010, pp. 111-118.

13. A. Krizhevsky, I. Sutskever, and G. E. Hinton, "Imagenet classification with deep convolutional neural networks," in Advances in neural information processing systems, 2012, pp. 1106-1114.

14. R. F. Nogueira, R. de AlencarLotufo, and R. C. Machado, "Fingerprint liveness detection using convolutional neural networks," IEEE Transactions on Information Forensics and Security, vol. 11, no. 6, pp. 1206- 1213, 2016.

15. T.-Y. Lin, A. Roy Chowdhury, and S. Maji, "Bilinear cnn models for finegrained visual recognition," in Proceedings of the IEEE International Conference on Computer Vision, 2015, pp. 1449-1457.

16. A. Ross and A. Jain, "Biometric sensor interoperability: A case study in fingerprints." Proc. Bio AW, LNCS 3086, Springer, 2004, pp. 134-145.

17. Arun Ross and Rohan Nadgir, "A Thin-Plate Spline Calibration Mode for Fingerprint Sensor Interoperability", IEEE Transactions On Knowledge And Data Engineering, Vol. 20, No. 8, August 2008.

18. Qian Zheng1, Ajay Kumar and Gang Pan, "Contactless 3D Fingerprin Identification Without 3D Reconstruction", IEEE conference on Computer Science and Security 2018.

\section{AUTHORS PROFILE}

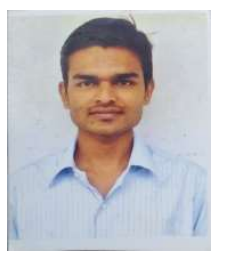

Sunil received the B Tech degree from Pune University, India, in 2016 . He is currently a final year M Tech student of Pimpri Chinchwad College of Engg. Pune University, India. He's area of research is Image processing.

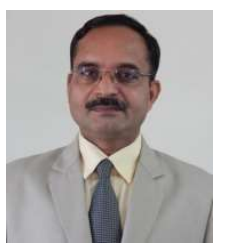

Dr.Kishor Kinage is professor at Pimpri Chinchwad college of engg, Pune, India. He's areas of Interest include Image Processing, Machine learning. 